\title{
Can illness be bright? Metaphor comprehension depends on linguistic and embodied factors
}

\author{
Pei Liu ${ }^{1}$, Louise Connell ${ }^{1}$ and Dermot Lynott ${ }^{1,2}$ \\ ${ }^{1}$ Department of Psychology, Lancaster University \\ ${ }^{2}$ Department of Psychology, Maynooth University
}

\begin{abstract}
Author Note
We have no conflicts of interest to declare. Correspondence concerning this article should be addressed to Louise Connell (1.connell@lancaster.ac.uk) or Dermot Lynott (dermot.lynott@,mu.ie)
\end{abstract}

Word count: 11171 


\begin{abstract}
Conceptual representation is both linguistic and embodied, at least when processing literal language. Here, we aim to demonstrate that metaphor processing is no exception. We investigated how the linguistic component (i.e., linguistic distributional frequency: the co-occurrence frequency of the constituent words in a metaphor) and the embodied component (i.e., how easy it is to simulate the concept: operationalized in a previous norming study) affect metaphor comprehension. Results show that ease of simulation contributes to metaphor processing in both shallow sensibility judgement and deep interpretation generation tasks, but has a larger effect on response times in deeper processing. Linguistic distributional frequency also contributes to metaphor processing in both tasks, but affects the speed of response only in shallow sensibility judgement. Specifically, distributional frequency acts as a linguistic shortcut, both to speed up responses to accept metaphors as sensible when the frequency is high, and to flag up potentially unsuccessful processing when it is low. Overall, these results support embodied views that emphasize the importance of both linguistic and embodied components according to task goals.
\end{abstract}

Keywords: metaphor processing, embodied simulation, linguistic distributional pattern, linguistic shortcut hypothesis

Word count: 11171 words 
Can illness be bright? Metaphor comprehension depends on linguistic and embodied factors

Metaphors add warmth and color to language, and can be understood so effortlessly by language users that their use does not register as an artifice. In linguistic terms, a metaphor is a figure of speech where a word or a phrase (the source) is applied to an object or an action (the target) to which it cannot be literally applied. For example, we can easily comprehend and frequently use the expression bright students even though a student is not an object to which the visual property of bright can be applied. Such metaphors are an essential part of language, not least in assisting us to express abstract concepts such as time, emotions and relationships (e.g., Boroditsky, 2001; Chan, Tong, Tan, \& Koh, 2013; Lakoff \& Johnson, 1999).

Traditionally, much research on metaphor comprehension has focused on identifying particular factors that could make a metaphor easier - and thus faster to understand, such as familiarity, conventionality and aptness (Blasko \& Connine, 1993; Bowdle \& Gentner, 2005; Cardillo, Schmidt, Kranjec, \& Chatterjee, 2010; Giora, 1997; L. L. Jones \& Estes, 2006; Pierce \& Chiappe, 2008; Roncero \& Almeida, 2014b). However, as we shall discuss below, these factors suffer from problems of theoretical specificity and empirical operationalization that make it difficult to draw clear conclusions about their roles in metaphor comprehension. Thus, it is necessary to study metaphor processing under a theoretical construct with better operationalized factors. In this paper, we look at the embodied/grounded perspective of conceptual representation, which suggests that language comprehension is an interactive conglomerate of activating linguistic symbols and simulating physical experiences. We propose how these components affect metaphor processing and investigate their roles with reference to how they 
relate to depth of processing during comprehension.

\section{Traditional Views of Metaphor Processing}

Several factors are often studied in research on metaphor processing, namely familiarity, conventionality and aptness, all of which have been demonstrated to affect the speed of metaphor comprehension. The graded salience hypothesis suggests that familiar or conventional metaphors are processed automatically because they enjoy high salience, compared with unfamiliar or novel metaphors (Blasko \& Connine, 1993; Giora, 1997, 2007; Glucksberg, Gildea, \& Bookin, 1982; Laurent, Denhières, Passerieux, Iakimova, \& Hardy-Baylé, 2006; Peleg, Giora, \& Fein, 2001). Moreover, conventionality could be a crucial factor in determining the mechanism with which metaphor is processed, according to an influential theory called the career of metaphor (Bowdle \& Gentner, 2005). In this theory, metaphors (e.g., My lawyer is a shark) first start out as novel or unconventional constructions and are processed by comparing the source concept (e.g., shark) with the target (e.g., lawyer) and identifying potential similarities to form the basis of the metaphoric meaning (e.g., aggressive). As a metaphor is used more often, it becomes conventionalized (e.g., aggressive becomes a conventional attribute for "shark") and the metaphor is processed by categorizing the target as a member of this conventional category (e.g., shark $\rightarrow$ *aggressive beings). In contrast, proponents of the theory of class inclusion suggest that it is aptness, rather than conventionality, that decides whether a metaphor is processed by categorization (Glucksberg 8 Haught, 2006b; Haught, 2014; L. L. Jones 8 Estes, 2005). That is, shark* can be categorized as a member of "aggressive beings" because the metaphor is apt (i.e., it works well), but a less apt metaphor would require comparing the target and source to find similarities.

Although these factors have been shown to affect metaphor processing to a 
certain degree, when it comes to understanding exactly how these three factors affect the process of metaphor comprehension, the problems are two-fold: first in their theoretical specificity, and second in their operationalization. In terms of theoretical specificity, these three factors are frequently confounded with one another and are internally inconsistent. Although some have suggested that familiarity and conventionality are two different theoretical constructs (Blasko \& Connine, 1993; Bowdle \& Gentner, 2005; Glucksberg et al., 1982; L. L. Jones \& Estes, 2005, 2006; Roncero \& Almeida, 2014a), they are often used interchangeably in the literature (Cardillo, Watson, Schmidt, Kranjec, \& Chatterjee, 2012; Giora, 1997; Giora \& Fein, 1999; Glucksberg \& Haught, 2006b; Lai \& Curran, 2013). Both terms are used to describe metaphors that at one extreme are entirely novel and have never been encountered before by participants, while at the other extreme are very common, conventional, and familiar to participants. Furthermore, aptness can also be confounded with familiarity and conventionality. Not only are familiarity and conventionality both dependent on usage patterns of a metaphor across a language, but aptness itself can also be associated with usage patterns. Only apt metaphors are likely to become conventionalized or familiar, as a metaphor that does not work well is unlikely to become widely used by speakers of a language. Hence, familiar/conventional metaphors are typically apt, whereas novel metaphors might be either apt or not. There is a core dependency between the factors that is not trivial to disentangle. Indeed, ratings of aptness and familiarity are highly correlated ( $r=.73-.82$ : Katz, Paivio, Marshark, \& Clark, 1988; Roncero \& Almeida, 2014a), as are ratings of aptness and corpus frequency counts of the metaphoric expression ( $r=.41-.57$ : Roncero \& Almeida, 2014b; Thibodeau \& Durgin, 2011). This core dependency means that we cannot investigate how these factors jointly or independently affect metaphor processing.

Moreover, the definitions of these critical factors are internally inconsistent. It 
is ambiguous whether familiarity/conventionality describe a metaphoric expression, or its metaphoric meaning. For example, familiarity is sometimes assumed to refer to how often people have encountered the metaphoric expression itself (e.g., how often is bright used to describe students? Blasko \& Connine, 1993; Bowdle \& Gentner, 2005; Cardillo et al., 2012; Glucksberg, 2003; Mashal, 2013), and sometimes to how accustomed people are to relating the expression to its metaphoric meaning (e.g., how often is bright understood to mean intelligent and quick-witted: Campbell \& Raney, 2015; Cardillo et al., 2010; Giora, 1997; Giora \& Fein, 1999; Katz et al., 1988; Peleg et al., 2001; Roncero \& Almeida, 2014a), but these are two very different and dissociable theoretical constructs. A particular linguistic expression might be encountered reasonably often but remain poorly understood (e.g., hard Brexit may have high expression familiarity but low meaning familiarity), or a metaphoric meaning might be encountered reasonably often via a different expression to the one supplied (e.g., bright solutions may have high meaning familiarity but low expression familiarity).

Because of the inconsistency in definition, these factors are not always consistently or rigorously operationalized in empirical studies that seek to examine their influence on metaphor comprehension. Typically, researchers measure each factor by asking participants to rate metaphors on a particular scale. Participants providing familiarity ratings are sometimes asked to rate the extent to which they have read or heard the expression in the past (Mashal, Faust, \& Hendler, 2005; Roncero \& Almeida, 2014a), sometimes to rate the familiarity of the ideas expressed in the metaphors (Blasko \& Connine, 1993; Campbell \& Raney, 2015; Katz et al., 1988), and at other times to rate the frequency of experience with both the expression and the meaning together (Cardillo et al., 2010). It is therefore unclear whether the reported effects of familiarity on metaphor processing times reflect experience at the word level (i.e., similar to word or phrase frequency) or experience 
with understanding the metaphoric meaning in question (i.e., experience in representing the metaphoric concept).

The same problem exists with the definition and operationalization of conventionality. In studies that test its empirical effects on metaphor processing, conventionality is sometimes operationalized as a function of the entire metaphoric expression by actually asking participants to rate familiarity rather than conventionality (e.g.: how familiar is the metaphor? Cardillo et al., 2010, 2012; Faust \& Mashal, 2007; Lai, Curran, \& Menn, 2009; Mashal et al., 2005), but sometimes as how often the metaphoric meaning is associated with the source (e.g., how conventional is it to use bright to mean intelligent?: Bowdle \& Gentner, 2005; Gentner \& Wolff, 1997; L. L. Jones \& Estes, 2006; Roncero \& Almeida, 2014a). While the former is confounded with familiarity, the latter operationalization has been challenged by findings that conventionality is context-dependent and cannot be defined for the source term independent of the target (Thibodeau \& Durgin, 2011). That is, just because Students can be bright is a conventional metaphor does not mean all metaphors that use bright as a source domain for intelligent and quick-witted (e.g., Solutions can be bright) are equally conventional. In short, it is unclear whether conventionality is a theoretically valid construct, and whether the reported effects of conventionality on metaphor processing are truly distinct from familiarity (which has its own problems, as discussed above).

Investigation of the importance of aptness faces a similar challenge. It has been ambiguously defined as either a very general, high-level quality or goodness of a metaphor and is often operationalized as such (Blasko \& Connine, 1993; Bowdle \& Gentner, 2005; Campbell \& Raney, 2015; Glucksberg \& Haught, 2006b; Katz et al., 1988), or as a much more low-level specification of how well the metaphoric meaning (e.g., intelligent and quick-witted) fits or overlaps with the target (e.g., students: Chiappe \& Kennedy, 1999; L. L. Jones \& Estes, 2005, 2006; Pierce \& Chiappe, 2008; 
Roncero \& Almeida, 2014b). Thibodeau and Durgin (2011), in a detailed examination of aptness ratings, argue that people are influenced by extraneous factors such as processing fluency when rating aptness and hence these ratings do not capture the theoretical construct they are meant to operationalize.

In summary, familiarity, conventionality and aptness have all been shown to affect metaphor processing. However, they have several theoretical and operational problems that mean they have limited utility in enhancing our understanding of what makes a metaphor easier to process. Rather than continue to vary and refine how these factors are conceptualized, we propose that a different approach is needed to seek clearer predictors of metaphor processing that (a) are theoretically and operationally distinct, and (b) are able to independently account for speed and accuracy performance in metaphor processing.

\section{Grounded Views of Language Processing}

Research in conceptual representation and language processing has tended to operate in parallel to that of traditional metaphor processing, and therefore takes quite a different perspective on how access to meaning takes place. Essentially, two components are employed in the mental representation of meaning when people process language (Barsalou, Santos, Simmons, \& Wilson, 2008; Borghi \& Binkofski, 2014; Connell \& Lynott, 2014b; Louwerse \& Jeuniaux, 2008; Lynott \& Connell, 2010; Vigliocco, Meteyard, Andrews, \& Kousta, 2009). The first component is linguistic and relies on the statistical, distributional pattern of how words co-occur across contexts. For example, the words bright and students occur together more often in language than do bright and illness, and such distributional frequency patterns have been shown to be powerful enough to capture many aspects of language processing, such as priming and reading times (Vigliocco et al., 2009), language acquisition from early infancy (Aslin, Saffran, \& Newport, 1998; Kirkham, 
Slemmer, \& Johnson, 2002), and even to some extent distinguishing words that relate to different perceptual modalities (Louwerse \& Connell, 2011). The second component of conceptual representation is embodied (also known as the grounded, sensorimotor or situated), which relies on the process of simulation; that is the partial reactivation of past perceptual, motor, affective, introspective and other experiences (Allport, 1985; Barsalou, 1999; Glenberg \& Gallese, 2012; Niedenthal, 2007). Evidence for embodied simulation includes shared activation between brain areas involved in perceptual or action experience and their equivalents in language comprehension. For example, reading sound-related words like "thunder" activates the auditory association cortex, and their processing is selectively impaired in patients with atrophy of the auditory association cortex (Bonner \& Grossman, 2012; Boulenger et al., 2008; Goldberg, Perfetti, \& Schneider, 2006; Hauk, Johnsrude, \& Pulvermüller, 2004).

Together, the linguistic and embodied components can explain language processing better than either alone (Andrews, Vigliocco, \& Vinson, 2009; Johns \& Jones, 2012; Louwerse \& Connell, 2011). In particular, linguistic distributional information provides a powerful tool for superficial language processing because the linguistic component is faster than the embodied simulation component (Barsalou et al., 2008; Louwerse \& Jeuniaux, 2008). In any language processing task, both linguistic and embodied components are activated but the activation of the linguistic component will peak before that of the embodied component (Barsalou et al., 2008). Therefore, Louwerse and Jeuniaux (2008) argued that people are more likely to rely on the embodied component when deeper processing is specifically cued in the task (Connell \& Lynott, 2014b); but people will be reliant upon the linguistic component to generate a good-enough approximation (Ferreira, Bailey, \& Ferraro, 2002) when shallow processing can suffice. Evidence for a speed advantage of the linguistic over the embodied component has been found in behavioral (Louwerse \& Connell, 2011; 
Santos, Chaigneau, Simmons, \& Barsalou, 2011), electrophysiological (Louwerse \& Hutchinson, 2012), and neuroimaging (Simmons et al., 2007) studies.

In line with these arguments, Connell and Lynott (2013) proposed that information from the linguistic component could act as a cognitive triage mechanism during language processing by providing a guide to whether it is worth expending effort on relatively costly embodied simulation (Connell, 2018). Since the co-occurrence of words in language tends to reflect the associations of objects, events, and ideas in the real world, the linguistic component can provide a rough and ready approximation of whether the embodied component will actually succeed in simulating a sensible meaning. Moreover, since activation in the linguistic component is faster than in the embodied component, the linguistic component has the potential to provide a shortcut by guiding task processing before the embodied component is fully engaged. If the linguistic component indicates that future processing is likely to fail or entail unnecessary costs (e.g., the words rarely co-occur in the same context and so their combined meaning might not be simulated successfully), then it could abandon the processing before any more cognitive effort is expended by the embodied component. On the other hand, if the linguistic component indicates that future processing is likely to succeed relatively easily (e.g., the words often co-occur in the same context and so their combined meaning can probably be simulated successfully), then it could either inform a response immediately (i.e., based on the linguistic shortcut alone) or allow the embodied component to continue developing a detailed simulation of meaning. Although the linguistic shortcut can provide a cognitive triage mechanism in any sort of cognitive task, it is more likely to form the basis of responses in circumstances where a detailed response is not required and a good-enough heuristic will therefore suffice, such as tasks that allow relatively shallow or superficial processing, or individual trials where deep and detailed processing has been abandoned. 
To test this proposal, Connell and Lynott (2013) asked participants to perform two tasks with novel noun-noun compounds (e.g., cactus beetle): a shallow processing task where participants judged if the compounds made sense, or a deep processing task where they decided if they could generate specific interpretations for them. The results showed that, consistent with linguistic shortcut predictions, the linguistic distributional frequency of the constituent words (i.e., how often cactus and beetle co-occurred) predicted both the likelihood of accepting versus rejecting a compound as sensible and the time course of both successful and unsuccessful processing in the shallow sensibility judgement task. However, in the deeper interpretation generation task, distributional frequency only predicted the likelihood of accepting versus rejecting a compound as interpretable and the timecourse of unsuccessful (i.e., abandoned) processing, but not the timecourse of successful processing that resulted in a detailed interpretation. In other words, while rejecting a noun-noun compound as nonsensical or uninterpretable needed only the linguistic shortcut to quickly flag up unsuccessful processing, the time needed to accept a compound as interpretable may require more detailed simulation which cannot be captured by the linguistic distributional frequency.

\section{Present Study}

Connell and Lynott's proposal can be applied directly to the study of metaphor processing, because the interplay of the linguistic and embodied components, and the role of the linguistic shortcut as a cognitive triage mechanism, operates in theory across all types of language comprehension. However, Connell and Lynott's study was unable to directly investigate the interplay between linguistic distribution and embodied simulation during language processing because it lacked at the time a direct measure of successful simulation (i.e. the ability to mentally represent the meaning of the phrase or sentence). The current study also 
faces the same obstacle in operationalizing how easy it is to generate successful simulation, because the meaning of a metaphor, by definition, cannot be accessed directly by looking at the literal meaning of its constituent words (Liu, Connell, \& Lynott, 2018). Furthermore, because simulations are unconscious mental representations that lose information when brought to conscious awareness (Connell \& Lynott, 2016), the ease of simulation cannot be directly rated as, for example, the ease of understanding a metaphor or of generating mental imagery. Therefore, in the present study, we used a new norming metric by Liu et al. (2018) that was explicitly designed to quantify how easy people find it to arrive at a mental representation of metaphoric meaning. It proposed three indirect measures of successful metaphor processing that, together, offer a proxy for ease of simulation: sensibility (How much sense does the sentence make if you read it in a story or heard it in conversation?), usability (How easy it would be for you to use the sentence in writing or in conversation?), and imaginability (How easy it is for you to imagine the concept described in the sentence?). Using principle components analysis, these three ratings scales were combined into a single ease of simulation measure that captured their common variance.

The ease-of-simulation measure is agnostic towards the content of the simulated representation. Instead, it measures the end-product of simulation - a complete and coherent representation of meaning - and uses it to quantify the effort of comprehension. If people find it easy to make sense of, use and imagine a metaphor, they would necessarily find the concept easy to simulate as well. Indeed, this ease-of-simulation measure can not only capture the majority of the variance (91\%) of the original subscales but also predict metaphor processing time better than any of the individual subscales (Liu et al., 2018), supporting the idea that it captures the underlying mechanism of conceptual representation. Thus, we expect it to be a powerful predictor of both speed and accuracy of metaphor processing in the 
current study as well.

Moreover, the grounded views of conceptual representation also predict that the linguistic component (i.e., linguistic distributional frequency (LDF) calculated from a large corpus) would be able to account for metaphor processing independently of the embodied component (i.e., ease of simulation). Although it remains a contentious issue how much the linguistic component contributes to the representation of meaning, it could be suggested that these two components are of distinct natures and perform different functions (Barsalou et al., 2008; Connell, 2018; Louwerse, Hutchinson, Tillman, \& Recchia, 2015; Riordan \& Jones, 2011). If our study can show that ease of simulation and LDF can each explain a unique, distinct portion of variance in performance of metaphor processing, this should provide strong support for the grounded approach of conceptual representation. Furthermore, as the cognitive triage mechanism suggests, the effects of these two factors should vary according to required depth of processing predicated by the tasks.

In the present paper, we examine the roles of the linguistic component (i.e., LDF calculated from a large corpus) and the embodied component (i.e., ease of simulation measure) in metaphor processing. There are several parallels between these two components and the factors that have traditionally been the focus of metaphor processing (i.e. familiarity, conventionality, and aptness). Both familiarity and conventionality are essentially measures of how often a metaphor has been previously experienced, and aptness too is strongly related to frequency of experience (Roncero \& Almeida, 2014b; Thibodeau \& Durgin, 2011). Our measure of LDF, which reflects how often the words in the metaphoric expression have been encountered in close proximity to one another (e.g., how frequently bright and students co-occur in language), is an objective metric of past experience with the source and target terms. The extent to which familiarity, conventionality, and 
aptness are based on frequency of experience is therefore captured by LDF. In addition, the notion of aptness - that is, how well the metaphor works - is closely tied to whether or not a mental representation of the metaphoric meaning can be successfully created. An apt metaphor that works well will result in a successful simulation of meaning (e.g., Students can be bright $\rightarrow$ "students can be intelligent and quick-witted"), whereas an inapt metaphor that does not work will not result in a coherent simulation (Illness can be bright $\rightarrow$ "Illness can be clever"). Our measure of ease of simulation (EoS) reflects the qualities that a complete and coherent simulation of meaning might be expected to have (i.e., it makes sense, is easy to imagine, and is easy to re-use: the components that make up the ease of simulation score). Therefore, EoS captures the extent to which aptness is based on successful representation of metaphoric meaning. Thus, in two experiments, we asked participants to process metaphors that systematically varied in LDF and EoS in a shallow task (Experiment 1: sensibility judgement task) or in a deep task (Experiment 2: interpretation generation task). Since the role of the linguistic and embodied components in language comprehension varies according to available resources, task demands, and processing goals (Connell \& Lynott, 2014b), their roles in metaphor processing will vary across experiments. Based on the operation of each component (Barsalou et al., 2008; Louwerse \& Connell, 2011; Louwerse \& Jeuniaux, 2008), and the operation of the linguistic shortcut mechanism (Connell, 2018; Connell \& Lynott, 2013), we propose the following hypotheses:

1. Simulation hypothesis: People will rely on the embodied component in both tasks. A metaphor that is easy to simulate will be accepted as sensible or interpretable more often. The effect of EoS on response time will be in opposite directions for "yes" and "no" responses. That is, it will take people less time to accept a metaphor that is easy to simulate, and less time to reject a metaphor (as nonsensical or uninterpretable) that is difficult to simulate 
(i.e., the harder a metaphor is to simulate, the quicker people will give up trying to process it). However, EoS should have a larger effect on accept ("yes") than reject ("no") response times because the latter reflects abandoned processing in the embodied component.

2. Linguistic distribution and linguistic shortcut hypothesis: People will also rely on the linguistic component in both tasks, where the linguistic shortcut will be used as a cognitive triage mechanism to identify processing that is unlikely to succeed and should be abandoned to avoid unnecessary costs. A metaphor with high LDF will be more likely to be accepted as sensible or interpretable than a metaphor with lower LDF (i.e., the less often words co-occur in language, the more likely people will be to abandon processing rather than engage in further, costly attempts at simulation). Furthermore, similar to the simulation hypothesis, the effects will be in opposite directions for "yes" and "no" responses: it will take people less time to accept a metaphor that has high LDF, and less time to reject a metaphor that has low LDF.

3. Depth of processing hypothesis: People will rely on EoS more for interpretation generation than for sensibility judgement because the former requires deeper processing and more detailed simulation. In contrast, people will rely on LDF more in shallow sensibility judgement, where "good enough" processing will suffice, than in deep interpretation generation.

In both experiments, we report how we determined our sample size, all data exclusions, all manipulations, and all measures in the study.

\section{Experiment 1: Sensibility Judgement Task}

In this study, participants were encouraged to process metaphors in a shallow way by judging whether or not the sentences made sense, where they made a forced 
choice between "yes" and "no". Sensibility judgement can be considered a relatively shallow form of language processing because it involves only a single yes/no response and participants are not required to specify any further details of how or why the sentence makes sense (Connell \& Lynott, 2013; Lynott \& Connell, 2010).

\section{Method}

Participants. Twenty-eight participants (five male and 23 female) took part in the study, same as Connell and Lynott (2013), all of whom were students at Lancaster University and native speakers of English with mean age of 19.1 years $(S D$ $=1.1$ ). Participation took approximately 20 minutes in exchange for $£ 3.00$ or course credits.

Materials. We used a total of 452 metaphoric sentences taken from Liu et al., (2016; see Table 1 for examples ${ }^{1}$ ). All sentences took the form "Noun can be adjective" (e.g., Student can be bright), and were composed of 113 perceptual adjectives (e.g., bright: Lynott \& Connell, 2009), each paired with four nouns (e.g.,

\footnotetext{
${ }^{1}$ All data and analysis scripts can be viewed through https://goo.gl/k1Q4jq. We used R (Version 3.5.0; R Core Team, 2018) and the R-packages abind (Version 1.4.5; Plate \& Heiberger, 2016), arm (Version 1.10.1; Gelman \& Su, 2018), BayesFactor (Version 0.9.12.4.2; Morey \& Rouder, 2018), bookdown (Version 0.7; Xie, 2016), broom (Version 0.4.4; Robinson, 2018), citr (Version 0.2.0; Aust, 2016), coda (Version 0.19.1; Plummer, Best, Cowles, \& Vines, 2006), data.table (Version 1.11.4; Dowle \& Srinivasan, 2018), ggplot2 (Version 2.2.1; Wickham, 2009), interplot (Version 0.1.5; Solt \& Hu, 2015), knitr (Version 1.20; Xie, 2015), lmeł (Version 1.1.17; Bates, Mächler, Bolker, \& Walker, 2015), lmerTest (Version 3.0.1; Kuznetsova, Brockhoff, \& Christensen, 2017), magrittr (Version 1.5; Bache \& Wickham, 2014), MASS (Version 7.3.50; Venables \& Ripley, 2002), Matrix (Version 1.2.14; Bates \& Maechler, 2018), MuMIn (Version 1.40.4; Bartoń, 2017), nlme (Version 3.1.137; Pinheiro, Bates, DebRoy, Sarkar, \& R Core Team, 2018), papaja (Version 0.1.0.9735; Aust \& Barth, 2018), psych (Version 1.8.4; Revelle, 2018), purrr (Version 0.2.5; Henry \& Wickham, 2018), and rcartocolor (Version 0.0.22; Nowosad, 2018) for all our analyses and the writing up of this manuscript.
} 
students/solutions/supply/illness) that were capable of eliciting metaphoric (i.e., non-literal) meanings, which varied systematically along the two critical dimensions of EoS and LDF.

Ease of simulation (EoS) scores for each sentence ranged from easy to difficult $(M=0.00, S D=1.00)$ and were taken from the norming study by Liu et al. (2017), where higher scores represent greater EoS. For example, both Students can be bright and Solutions can be bright were considered relatively easy to simulate, whereas both Illness can be bright and Supply can be bright were considered relatively difficult to simulate.

Linguistic distributional frequency (LDF) for each sentence ranged from low to high $(M=2.95, S D=0.97)$, and was calculated as the log of the summed bito five-gram frequencies of the sentence's noun and adjective in the Google Web1T Corpus (Brants \& Franz, 2006). To take the metaphor Students can be bright as an example, the LDF was the sum of the frequencies of "student ... bright" and "bright ... student" with zero, one, two, and three intervening words. Frequencies were $\log$ transformed as $\log _{10}$ (frequency +41$)$ where 41 is the lowest non-zero frequency in the item set (Connell \& Lynott, 2013). Within the four metaphors created for each adjective, LDF varied independently of EoS (see Table 1). For example, both Students can be bright and Supply can be bright had relatively high LDF, whereas both Illness can be bright and Solutions can be bright had lower LDF.

The sentences were split into four lists of 113 items each, where each adjective appeared only once per list, and the distribution of easy/difficult to simulate and high/low LDF was approximately equal across lists $\left(\right.$ EoS: $F_{(3,440)}=1.70, p=.166$; $\left.\mathrm{LDF}: F_{(3,440)}=0.43, p=.734\right)$. Each participant saw only one list.

Procedure. There were five practice trials (whose results were not recorded or analyzed) before the 113 test trials. Participants read one sentence in each trial 
Table 1

Sample metaphors, and their scores for EoS

and $L D F$

\begin{tabular}{lcc}
\hline Metaphor & EoS & LDF \\
\hline Illness can be bright. & -1.32 & 2.95 \\
Supply can be bright. & -1.02 & 3.72 \\
Solutions can be bright. & 1.41 & 3.11 \\
Students can be bright. & 1.84 & 4.08 \\
Minutes can be lukewarm. & -1.49 & 3.31 \\
Scheme can be lukewarm. & -0.78 & 1.61 \\
Supporters can be lukewarm. & 0.69 & 2.69 \\
Reaction can be lukewarm. & 1.03 & 3.45 \\
\hline
\end{tabular}

and decide whether or not the sentence made sense, based on oral and verbal instructions. All trials had the same structure (see Figure 1). Participants first saw a fixation cross for 1000 milliseconds (ms), followed by the noun for $500 \mathrm{~ms}$, followed by the phrase "can be" for $500 \mathrm{~ms}$, and then followed by the adjective. The adjective remained on the screen until participants made a response. Participants pressed either the comma key (",") if they judged that the sentence made sense; or the full stop key (".") if they judged that it did not make sense. They were allowed to make their response naturally, using the finger(s) most comfortable for them. The response could be made without a time limit; and participants were told explicitly that there were no right or wrong answers to the question. Both the response decision ("yes" to accept the metaphor as sensible; or "no" to reject the metaphor as nonsensical), and response time in milliseconds (RT) from onset of the adjective, were recorded as dependent variables. 


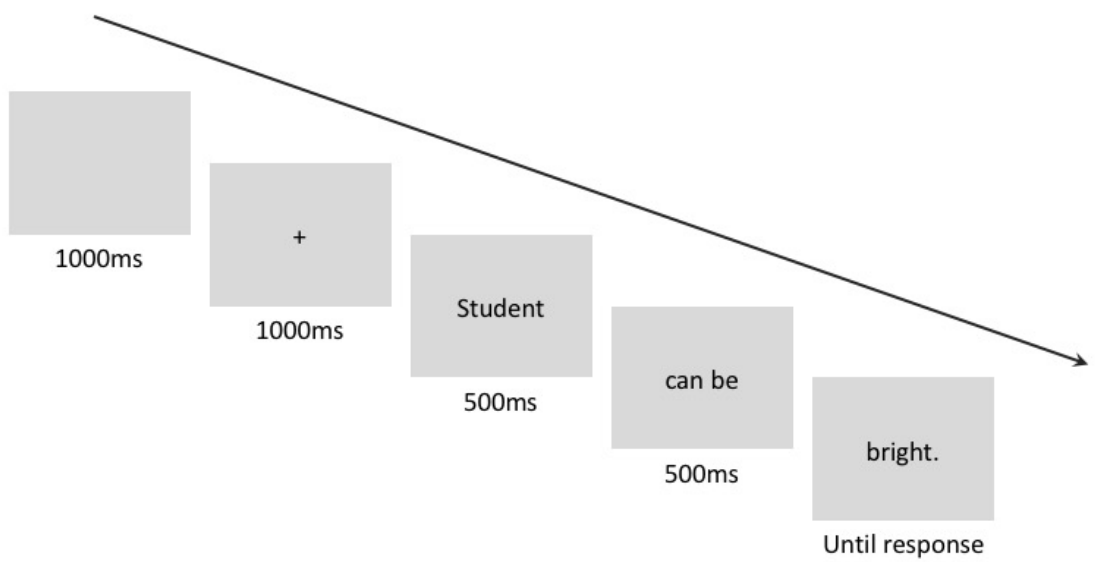

Figure 1. Trial structure in the sensibility judgement task.

Design and analysis. Response decisions were analyzed in a mixed effects logistic regression (binomial distribution with logit link), with the dependent variable of response (coded as 1 for "yes", accepting the metaphor as sensible; and 0 for "no", rejecting the metaphor as nonsensical), crossed random-intercept effects of participants and items, ${ }^{2}$ and fixed variables of LDF, EoS, and their interaction. Both LDF and EoS scores were centred before analysis.

Response times (RTs) were analyzed using mixed effects linear regressions, firstly in an omnibus analysis with crossed random-intercept effects of participants and items, ${ }^{3}$ and fixed variables of response, LDF, EoS, and their interactions.

\footnotetext{
${ }^{2}$ The inclusion of participants as a random factor improved model fit above the empty model, $\chi_{(1)}^{2}$ $=427.39, p<.001$, as did the inclusion of items as a crossed random factor above the participants-only model, $\chi_{(1)}^{2}=456.76, p<.001$.

3 The inclusion of participants as a random factor improved model fit above the empty model, $\chi_{(1)}^{2}$ $=853.15, p<.001$. The inclusion of items as a crossed random factor did not improve model fit above the participants-only model $\chi_{(1)}^{2}=0.00, p=.998$. However, in order to keep the models
} 
Secondly, we ran separate analyses on acceptance ("yes") and rejection ("no") responses because we expected the effects of the fixed variables to be in opposite directions for different response decisions. We initially considered the inclusion of word length and lexical frequency (i.e., sum of the log frequencies of nouns and adjectives, retrieved from Elexicon separately: Balota et al., 2007, http://elexicon.wustl.edu), but they were excluded from final analyses because their zero-order correlations with RT were near zero (see Supplementary Materials).

\section{Results and Discussion}

All participants had mean response times within 3SD of the overall mean and so all were included in analysis. Two trials were removed because of motor error (RT $<200 \mathrm{~ms})$. Furthermore, individual trials with RT more than three standard deviations from each participant's mean per response decision were removed as outliers: $1.33 \%$ of "yes" responses and $2.20 \%$ of "no" responses.

Response decision. Among 3105 valid trials, 1413 (45.51\%) were accepted as sensible ("yes" responses) and 1692 (54.59\%) were rejected as nonsensical ("no" responses). As predicted, logistic regression showed that EoS had a positive effect on the response decision, $z_{(1,3101)}=24.60, p<.001, b=1.253,95 \% \mathrm{CI}=[1.153,1.353]$. As EoS increased by one unit, the odds of accepting a metaphor as sensible (as opposed to rejecting it as nonsensical) increased 3.501 times. In contrast, LDF had a negative effect on the response decision, $z_{(1,3101)}=-2.02, p=.043, b=-0.093,95 \%$ $\mathrm{CI}=[-0.183,0.003]$, meaning that each increased unit of LDF made the decision to accept a metaphor as sensible less likely (odds ratio $=0.911$ ). Since this partialled

consistent, both participants and items were included as crossed random factors in the linear models of RT as well as in the logistic models of response decision. It should be noted that analyses of RT with participant-only models did not substantially change results; these analyses are reported in supplementary materials. 
relationship between response decision and LDF in the regression model was the inverse of their unpartialled relationship outside the regression model (i.e., mean LDF was higher for "yes" than "no" responses: Supplementary Materials), it suggests that net suppression was present in the model. ${ }^{4}$ That is, the shared error variance between LDF and EoS was effectively hiding the real relationship between LDF and response decision, such that the net effect of LDF in logistic regression was to enhance the effect of EoS by suppressing its unhelpful error variance (J. Cohen, Cohen, West, \& Aiken, 2003).

In order to establish the true relationships between response decision and our independent variables, we therefore removed the shared variance between LDF and EoS (currently correlated at $r=.27$ ) by orthogonalizing the variables. We used principal components analysis (PCA) to rotate the two original variables into two orthogonal components, which (rather than the traditional PCA use of dimension reduction) allowed us to disentangle two correlated variables without losing any information (Connell \& Lynott, 2014a; Glantz \& Slinker, 1990). Using varimax rotation with Kaisar normalization on a covariance matrix, Component 1 corresponded to $\operatorname{EoS}(r=.99)$ and Component 2 corresponded to LDF $(r=.99)$. These two orthogonalized variables accounted for $100 \%$ of the original variance while themselves were uncorrelated (i.e., $r=.00$ ). We re-ran the logistic regression with these orthogonalized variables and obtained results as follows (Table 2).

Logistic regression with these orthogonalized variables showed that EoS had a positive effect on response decision (Table 2). As the orthogonalized EoS increased by one unit, the odds of accepting a metaphor as sensible increased 3.421 times. Moreover, the effect of the orthogonalized LDF was also positive, but only as a weak

\footnotetext{
${ }^{4}$ A logistic regression model with linguistic associative frequency as the only fixed factor confirmed this possibility as it returns a positive coefficient, $t_{(1,3103)}=4.03, p<.001, b=0.36,95 \% \mathrm{CI}=$ $0.19-0.54$.
} 
Table 2

Logistic mixed-effect regression of response decision in Experiment 1

\begin{tabular}{lrrr}
\hline & $b$ & $95 \%$ CI & $p$ \\
\hline Intercept & -0.25 & $-0.62-0.13$ & .198 \\
EoS & 1.47 & $1.34-1.60$ & $<.001 * * *$ \\
LDF & 0.11 & $0.01-0.21$ & $.027 *$ \\
EoS x LDF & -0.00 & $-0.11-0.10$ & .946 \\
\hline Note. $\dagger$ means $p<.10 ; *$ means $p<.05 ;$ \\
$* *$ means $p<.01 ; * * *$ means $p<.001$. \\
$b$ is non-standardised regression coefficient. \\
Both predictors orthogonalized.
\end{tabular}

trend. This time, as the orthogonalized LDF increased, the odds of accepting a metaphor as sensible increased slightly (1.084 times) as well.

In other words, when people were asked to make a relatively shallow judgement about whether or not a metaphor made sense, their yes/no decision was influenced predominantly by EoS (easy-to-simulate metaphors were more likely to be judged as sensible) but also by the LDF of the words used in the metaphor (frequently co-occurring words were slightly more likely to be judged as sensible; infrequently co-occurring words were slightly more likely to be rejected as nonsense). Both the linguistic and embodied components played a role in metaphor processing.

Response time. As in the logistic regression of response decision, linear analysis of response times ( $M=1139 \mathrm{~ms}, S D=587 \mathrm{~ms}$ ) also produced evidence of net suppression (see supplementary materials for details). For that reason, and to maintain the independence between the predictors in all our models, we 
Table 3

Omnibus linear mixed-effect model of RT in Experiment 1

\begin{tabular}{lrrr}
\hline & $b$ & $95 \%$ CI & $p$ \\
\hline Intercept & 1129.66 & $1011.04-1248.29$ & $<.001 * * *$ \\
EoS & 67.06 & $41.76-92.36$ & $<.001 * * *$ \\
LDF & 14.76 & $-9.81-39.32$ & .239 \\
Response & 0.76 & $-36.86-38.38$ & .969 \\
EoS x LDF & 24.39 & $1.18-47.61$ & $.040 *$ \\
EoS x response & -181.55 & $-218.91--144.17$ & $<.001 * * *$ \\
LDF x response & -38.54 & $-74.48--2.61$ & $0.036 *$ \\
EoS x LDF x response & -32.50 & $-67.07-2.08$ & $0.066 \dagger$ \\
\hline
\end{tabular}

Note. $\dagger$ means $p<.10 ;{ }^{*}$ means $p<.05$;

** means $p<.01 ; * * *$ means $p<.001$.

$b$ is non-standardised regression coefficient.

Both predictors orthogonalized.

orthogonalized the variables per response type. Table 3 shows full results of the omnibus analysis across all responses. Overall, EoS had a positive effect on RT, meaning that response times were generally slower for easy-to-simulate metaphors. However, EoS also interacted negatively with response decision, suggesting that the direction of the EoS effect differed by response type. LDF had no overall main effect, but interacted with response decision to indicate that the direction of LDF differed for "yes" and "no" RTs.

Since we had separate hypotheses for "yes" and "no" RTs, we divided the dataset by response decision and analyzed their RT separately. Results are given in Table 4. For "yes" responses (i.e. metaphors that were accepted as sensible; RT: $M$ 
Table 4

Regression model on $R T$ in Experiment 1 per response decision

\begin{tabular}{lrrrrrrr}
\hline & \multicolumn{3}{c}{ Yes } & & \multicolumn{2}{c}{ No } \\
\cline { 2 - 3 } & \multicolumn{1}{c}{$b$} & $95 \%$ CI & $p$ & $b$ & $95 \%$ CI & $p$ \\
\hline Intercept & 1172.16 & $1051.79-1292.52$ & $<.001 * * *$ & 1155.41 & $1010.94-1299.88$ & $<.001 * * *$ \\
EoS & -134.88 & $-161.98--107.79$ & $<.001 * * *$ & 72.28 & $47.27-97.29$ & $<.001 * * *$ \\
LDF & -27.52 & $-53.10--1.93$ & $0.036 *$ & 17.72 & $-6.40-41.83$ & 0.152 \\
EoS x LDF & -13.00 & $-37.90-11.91$ & 0.307 & 23.33 & $0.51-46.14$ & $0.046 *$ \\
\hline
\end{tabular}

Note. $\dagger$ means $p<.10 ; *$ means $p<.05$,

** means $p<.01 ; * * *$ means $p<.001$.

$b$ is non-standardised regression coefficient.

Both predictors orthogonalized per response decision.

$=1150 \mathrm{~ms}, S D=589 \mathrm{~ms}$ ), EoS had the predicted negative effect, such that the easier a metaphor was to simulate, the less time people took to accept it as sensible Table 4. LDF also had a negative effect on acceptance times as predicted. That is, the more often the words in a metaphor co-occurred in language, the faster people were to accept it as sensible. Figure 2 shows the standardized coefficients of each variable on RT per response decision.

For "no" responses (metaphors rejected as nonsensical; RT: $M=1114 \mathrm{~ms}, S D$ $=603 \mathrm{~ms}$ ), the effects ran in the opposite direction (Table 4). As predicted, EoS had a positive effect on RT, meaning that people were faster to reject metaphors that were normally regarded as difficult to simulate. LDF had no significant main effect but did positively interact with EoS, such that the effect of EoS was enhanced when LDF was high, but was reduced when LDF was low. Figure 3 shows the effects 


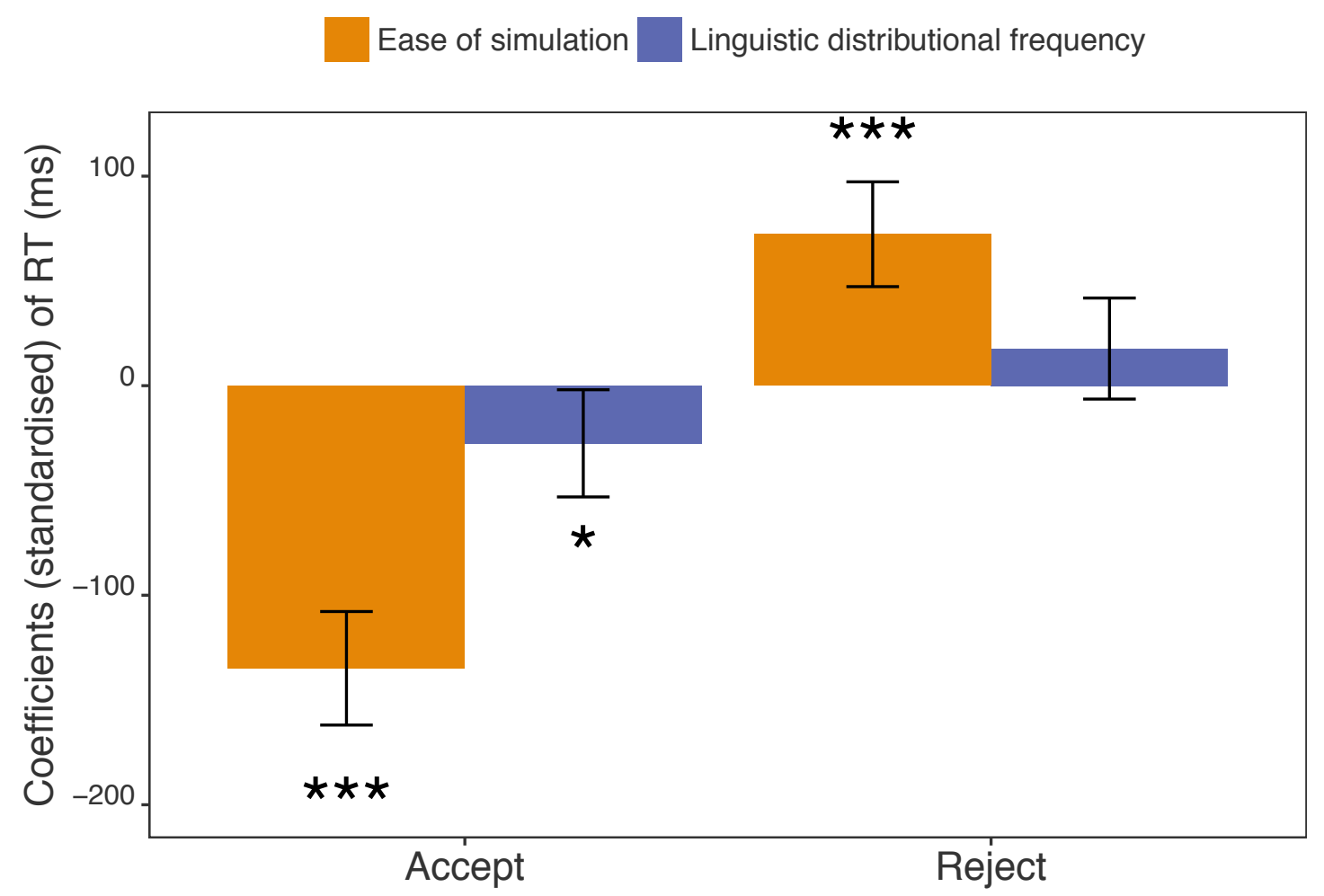

Figure 2. Effects of EoS and LDF, expressed as standardized regression coefficients, on acceptance and rejection times for sensibility judgement. Error bars represent 95\% confidence intervals, and asterisks represent the significance of directional predictions where specified $\left({ }^{* * *} p<.001,{ }^{* *} p<.01,{ }^{*} p<.05\right)$.

of EoS on the RT of reject ("no") responses as LDF increased. Overall, people were faster to reject a metaphor as nonsensical when its words rarely co-occurred and its meaning was difficult to simulate, and slower to reject metaphors whose words often co-occurred but whose meaning was generally considered easy to simulate.

Summary. Overall, results showed that people relied on both the embodied and linguistic components when judging metaphor sensibility. Following our simulation hypothesis, metaphors whose meanings were easy to simulate (e.g., Students can be bright; Solutions can be bright) were more likely to be accepted as sensible and were accepted more quickly, whereas metaphors whose meanings were 


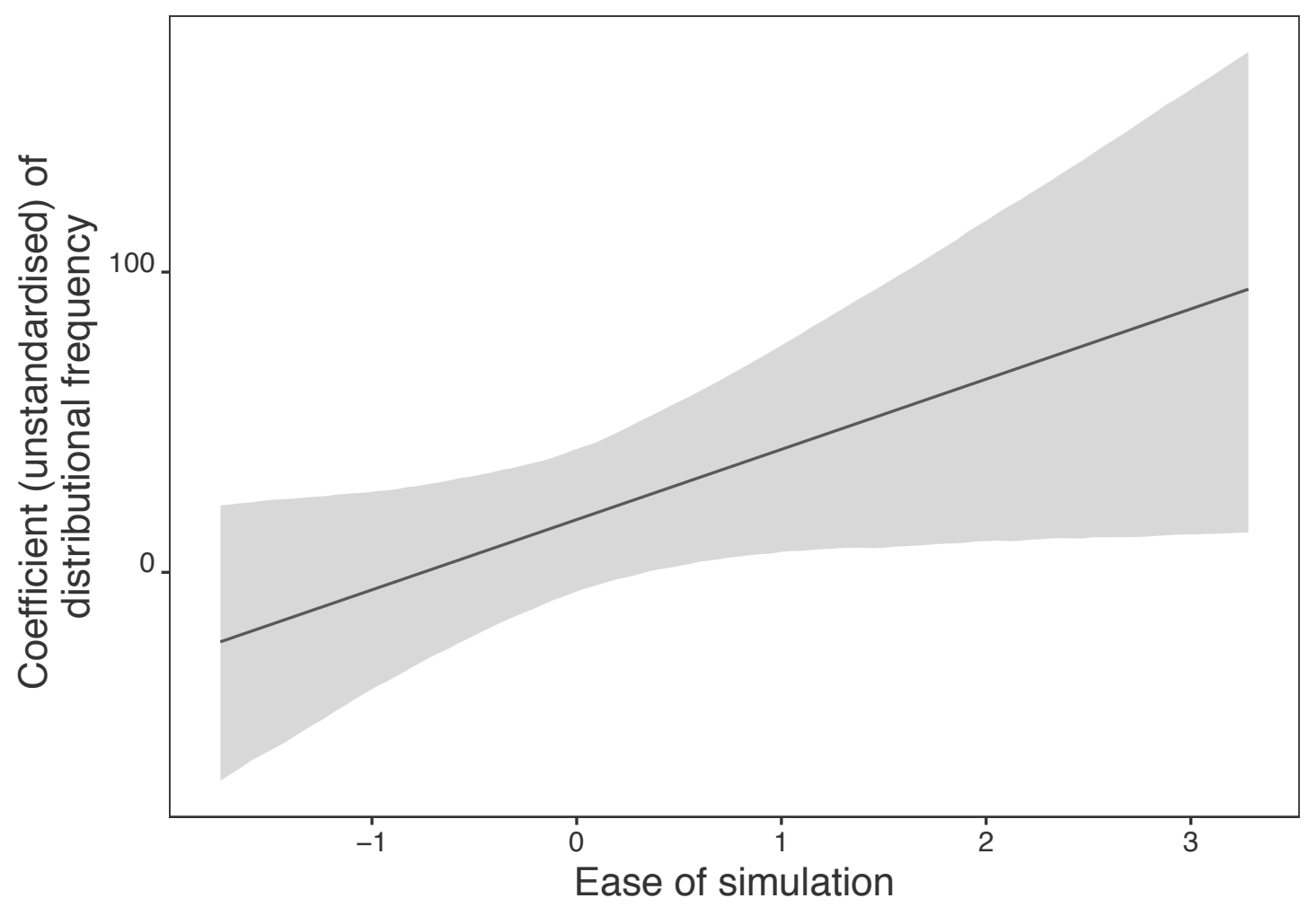

Figure 3. Change in the coefficients of LDF as EoS increased when people rejected metaphors as nonsensical. The shaded area indicates the $95 \%$ confidence interval.

typically regarded as difficult to simulate (e.g., Supply can be bright; Illness can be bright) were rejected more quickly. Moreover, EoS had a greater effect on metaphor acceptance times ("yes" $b=-134.88$ ) than on rejection times ("no" $b=72.28$ ), as predicted, due to the fact that acceptance times reflect simulation that has successfully completed in the embodied component whereas rejection times reflect simulation attempts that have been abandoned. In addition, as predicted by the linguistic distributional hypothesis, metaphors whose words often co-occurred in context (e.g., Students can be bright; Supply can be bright) were more likely to be accepted as sensible (although only marginally) and were accepted more quickly, while metaphors whose words rarely co-occurred (e.g., Illness can be bright) were not rejected directly. Instead, LDF moderated the effect of EoS (i.e. the effect of 
EoS was reduced when the LDF was low), which suggested that people rejected the metaphors as nonsensical based on their low LDF, without further processing in the embodied component. This pattern of findings supports the idea that the linguistic shortcut was used as a cognitive triage mechanism during metaphor comprehension to identify processing that was unlikely to succeed (i.e., produce a coherent simulation of meaning) and should therefore be abandoned rather than wasting resources on further processing.

\section{Experiment 2: Interpretation Generation Task}

In this study, participants were encouraged to process metaphors in a relatively deep way by completing an interpretation generation task. As they read each metaphor sentence, participants tried to think of a possible meaning for the sentence. If they could think of a meaning, participants were required to specify their interpretation. Interpretation generation can therefore be considered as a deeper form of metaphor processing than sensibility judgement because it requires participants to represent a particular meaning in detail (Connell \& Lynott, 2013; Lynott \& Connell, 2010). All metaphors were the same as in Experiment 1, but since participants would be engaging in deeper processing when generating interpretations than when simply judging sensibility, we expected they would be more likely to successfully simulate a meaning. Thus, we predicted a higher acceptance rate (i.e., more "yes" responses) in this study than in Experiment 1 (as per Connell \& Lynott, 2013), as well as our hypotheses earlier outlined.

\section{Method}

Participants. Forty native speakers of English (11 males and 29 females; age $\mathrm{M}=19.65, \mathrm{SD}=2.08$ ) were recruited from the same population as Experiment 
1 to match the coefficient of variation in Experiment 1. Participation took approximately 20 minutes in exchange for $£ 3.00$ or course credits.

Materials. Same as in Experiment 1.

Procedure. The procedure was identical to Experiment 1, except that instructions asked participants to come up with a meaning for each sentence, and to be clear and specific in their meanings. The trial structure was the same as Experiment 1 (see Figure 1) up to the point where participants were required to make a decision. In this study, if they pressed the comma (",") key to indicate that they could think of a meaning for the metaphor, they would be presented with a blank screen where they could type in the meaning just generated; if they pressed the full stop (".") key to indicate that they could not think of a meaning, they would proceed to the next trial.

The item lists from Experiment 1 were split in half pseudo-randomly while preserving the equal distribution of items along the dimensions of EoS and LDF, in order to reduce the possibility of fatigue, resulting in 8 lists of 56-57 items each. As before, each participant saw only one list. The whole task took approximately 20 minutes to complete and participants were given one break halfway through.

Design and analysis. Data were analyzed in the same way as in Experiment 1. Mixed effects logistic regression of response decisions contained crossed random-intercept factors of participants and items, as did mixed effects linear regressions of response times. ${ }^{5}$ As in Experiment 1, there was evidence of net

\footnotetext{
${ }^{5}$ In logistic regression, the inclusion of participants as a random factor improved model fit above the empty model, $\left.\chi_{(1)}^{2}=368.76, p<.001\right)$, as did the inclusion of items as a crossed random factor, $\chi_{(1)}^{2}=156.50, p<.001$. In linear regressions, the inclusion of participants as a random factor improved model fit above the empty model $\chi_{(1)}^{2}=638.85, p<.001$, although the inclusion of items as a crossed random factor did not improve model fit above the participants-only model, $\chi_{(1)}^{2}=0.00, p=1.00$. However, as in Experiment 1, both participants and items were included as
} 
suppression in the regression models due to the shared error variance between predictors (see supplementary materials for details). For that reason, and to maintain consistency in predictors between models and experiments, we therefore report the analysis using orthogonalized variables of EoS and LDF.

\section{Results and Discussion}

All participants had mean response times within 3SD of the overall mean so none were excluded on that criterion. We also identified accept ("yes") trials with invalid interpretations, namely those that were: blank or single characters,

participant-flagged errors (e.g., "mistake" or "don't know"), simple repetition of the original metaphor (e.g., Students can be bright $\rightarrow$ "students are bright"), replacement of the adjective with a synonym or an adjective that the researchers judged to be nonsensical in context (e.g., Borders can be deafening $\rightarrow$ "borders can be loud"; Economy can be freezing $\rightarrow$ "The economy can be dry"), and clear misreading of words (e.g. Charge can be rustling $\rightarrow$ "coins can be heard to make a [rustling] sound in pockets", consistent with misreading charge as change). Two participants were excluded from analysis for providing more than $50 \%$ invalid interpretations. Amongst the remaining participants, $2.33 \%$ of interpretations (31 trials) were identified as invalid and removed from analysis. No trials were removed due to motor error, but RTs more than 3 standard deviations from each participants' mean per response decision were removed as outliers: $2.10 \%$ of "yes" responses and $2.00 \%$ of "no" responses.

Response decision. Among 2103 valid trials, 1302 (61.91\%) were accepted as interpretable ("yes" responses) whereas 801 (38.09\%) were rejected as crossed random factors in the linear models of RT as well as in the logistic models of response decision in order to keep the analyses consistent. Analyses of RT with participant-only models did not substantially alter results, and are reported in supplementary materials. 
Table 5

Logistic mixed-effect regression of response decision in Experiment 2

\begin{tabular}{lrrr}
\hline & $b$ & $95 \%$ CI & $p$ \\
\hline Intercept & 0.96 & $0.42-1.50$ & $<.001 * * *$ \\
EoS & 1.53 & $1.35-1.71$ & $<.001 * * *$ \\
LDF & 0.34 & $0.20-0.48$ & $<.001 * * *$ \\
EoS x LDF & -0.01 & $-0.15-0.13$ & 0.887 \\
\hline Note. $\dagger$ means $p<.10 ; *$ means $p<.05 ;$ \\
** means $p<.01 ; * * *$ means $p<.001$. \\
Both predictors orthogonalized.
\end{tabular}

uninterpretable ("no" responses). Logistic regression showed that both EoS and LDF had a positive effect on response decision (see Table 5). For every unit of increase in EoS, the odds of accepting a metaphor as interpretable increased 2.826 times; and for every unit of increase in LDF, it increased 1.286 times.

In short, when people were asked to make a relatively deep judgement about whether they could think of a meaning for a metaphor, their yes/no decision was influenced by both EoS (easy-to-simulate metaphors were more likely to be interpretable) and by the LDF of the words used in the metaphor (frequently co-occurring words were more likely to give rise to an interpretable metaphor). Hence, both the linguistic and embodied components played a role in metaphor processing, as predicted.

Response time. Linear regression of RT across all responses $(M=2837 \mathrm{~ms}$, $S D=2469 \mathrm{~ms}$ ) found no overall effect of EoS (see Table 6). However, EoS interacted negatively with response decision, indicating the effect of EoS for "yes" was opposite 
Table 6

Omnibus linear mixed-effect model of RT in Experiment 2

\begin{tabular}{lrrr}
\hline & $b$ & $95 \%$ CI & $p$ \\
\hline Intercept & 2796.78 & $2341.82-3251.76$ & $<.001 * * *$ \\
EoS & 125.28 & $-20.79-271.35$ & $.093 \dagger$ \\
LDF & 44.47 & $-97.02-185.95$ & .538 \\
Response & 58.38 & $-143.64-260.40$ & .571 \\
EoS x LDF & -34.60 & $-167.90-98.71$ & .6111 \\
EoS x response & -589.63 & $-774.55--404.70$ & $<.001 * * *$ \\
LDF x response & -13.52 & $-193.60-166.55$ & .883 \\
EoS x LDF x response & 4.82 & $-166.13-175.76$ & .956 \\
\hline
\end{tabular}

Note. $\dagger$ means $p<.10 ;{ }^{*}$ means $p<.05$;

** means $p<.01 ; * * *$ means $p<.001$.

Both predictors orthogonalized.

to that for "no" responses. LDF did not have any significant effects or interactions.

As in Experiment 1, we had separate hypotheses for "yes" and "no" RTs and so analysed them separately; results are shown in Table 7. For "yes" responses (i.e. accepting metaphors as interpretable; RT: $M=3083 \mathrm{~ms}, S D=2638 \mathrm{~ms}$ ), EoS had a negative effect, as predicted, meaning that people were faster to accept a metaphor as interpretable when it was typically considered easy to simulate compared to difficult to simulate. LDF did not affect the speed of interpretation, nor was there an interaction (Figure 4).

For "no" responses that rejected the metaphor as uninterpretable (RT: $M=$ $2436 \mathrm{~ms}, S D=2105 \mathrm{~ms}$ ), EoS had a positive effect on RT: as predicted, people were faster to reject a metaphor as uninterpretable when it was normally considered 


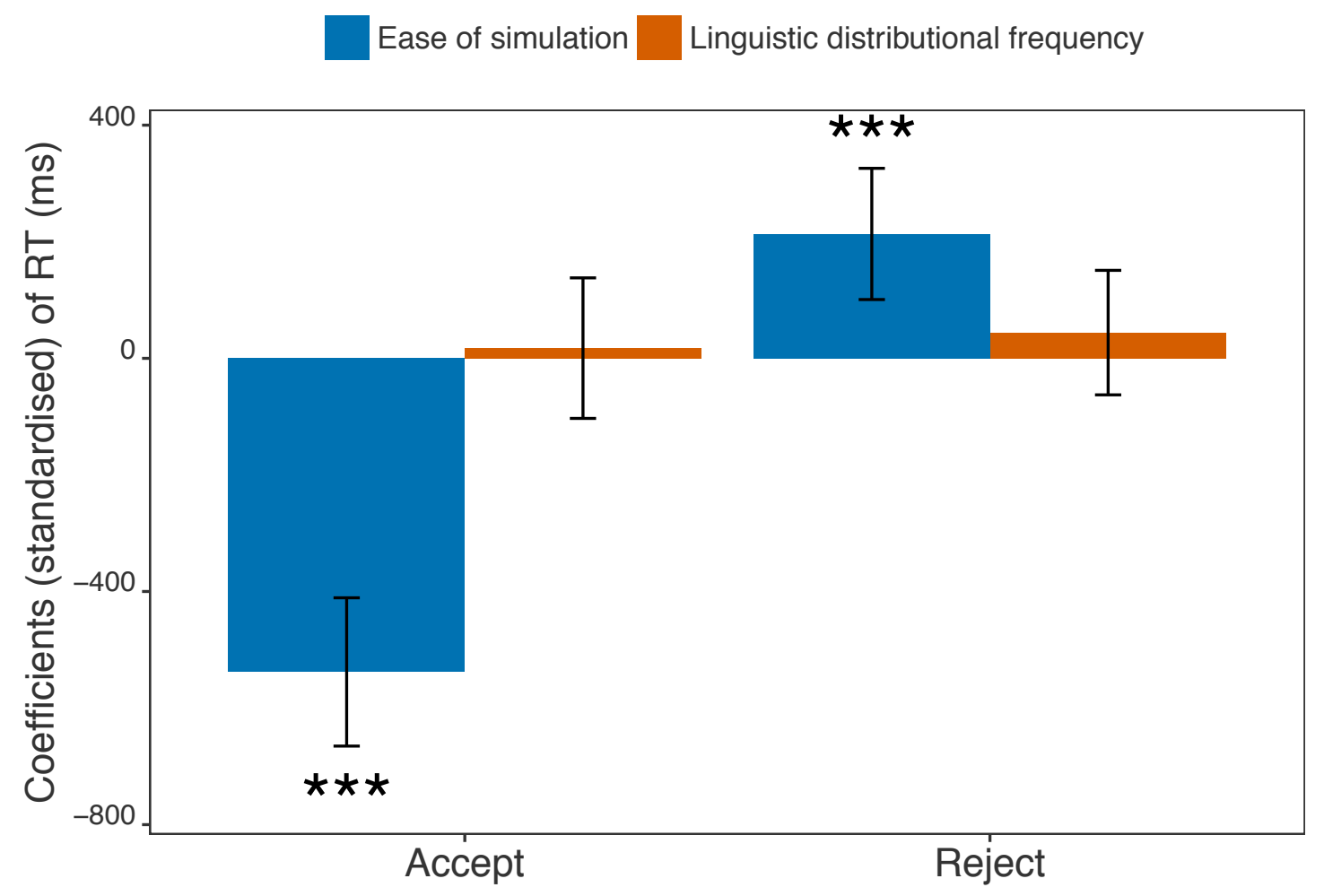

Figure 4. Effects of EoS and LDF, expressed as standardised regression coefficients, on acceptance and rejection times for interpretation generation. Error bars represent $95 \%$ confidence intervals, and asterisks represent the significance of directional predictions where specified $\left(* * * p<.001,{ }^{* *} p<.01,{ }^{*} p<.05\right)$.

difficult to simulate. LDF did not affect rejection speed, nor did it interact with EoS (Figure 4).

Summary. Results of Experiment 2's interpretation generation task were similar to those of Experiment 1's sensibility judgement, in showing that people relied on both the embodied and linguistic components when processing metaphors. EoS affected both the likelihood and processing speed of generating an interpretation for a metaphor, supporting the simulation hypothesis: the easier it was to simulate the meaning of a particular metaphor, the more likely it was to be interpreted and the faster people accepted it as interpretable. Conversely, metaphors 
Table 7

Regression model on $R T$ in Experiment 2 per response decision

\begin{tabular}{lrrrrrrrr}
\hline & \multicolumn{9}{c}{ Yes } & & \multicolumn{2}{c}{ No } \\
\cline { 2 - 3 } & \multicolumn{1}{c}{$b$} & $95 \%$ CI & $p$ & $b$ & $95 \%$ CI & $p$ \\
\hline Intercept & 2961.19 & $2507.85-3414.54$ & $<.001 * * *$ & 3245.15 & $2507.68-3982.63$ & $<.001 * * *$ \\
EoS & -538.01 & $-665.18--410.83$ & $<.001 * * *$ & 213.38 & $100.81-325.94$ & $<.001 * * *$ \\
LDF & 17.48 & $-103.06-138.02$ & 0.776 & 44.11 & $-62.70-150.91$ & 0.419 \\
EoS x LDF & -34.12 & $-149.71-81.46$ & 0.563 & -56.58 & $-158.13-44.98$ & 0.276 \\
\hline
\end{tabular}

Note. $\dagger$ means $p<.10 ; *$ means $p<.05$,

** means $p<.01 ; * * *$ means $p<.001$.

Both predictors orthogonalized per response decision.

whose meanings were difficult to simulate were rejected as uninterpretable relatively quickly. As predicted, EoS had a greater effect on metaphor acceptance times ("yes" $b=-538.00)$ where simulation was ultimately successful, than on rejection times ("no" $b=213.38)$ where processing in the embodied component was abandoned. Following the linguistic distributional hypothesis, metaphors whose words often co-occurred in context (e.g., Students can be bright) were more likely to be accepted as interpretable than metaphors whose words rarely co-occurred (e.g., Solution can be bright). The effects of LDF did not carry over into interpretation times, however, unlike in Experiment 1's sensibility judgement times. Hence, support for the use of the linguistic shortcut as a cognitive triage mechanism in deep metaphor processing is limited to its effect on the likelihood of interpretation. We examine such task differences further in cross-experiment analyses. 


\section{Cross-Experiment Analyses}

Since some of our hypotheses concern differences between shallow and deep processing tasks, we conducted a meta-analysis of the data from Experiments 1 and 2 in order to compare effect sizes between sensibility judgement and interpretation generation tasks.

\section{Method}

The data from Experiments 1 and 2 were combined for analysis: response decision in logistic mixed effects regression, and response time in linear mixed effects regression. All analyses used the same models with orthogonalized variables that were previously employed in separate experiments, this time including an additional fixed predictor variable of task (coded 0 for sensibility judgement, 1 for interpretation generation) that interacted with all other predictors.

\section{Results and Discussion}

Response decision. Results are shown in Table 8. The likelihood of accepting versus rejecting a metaphor varied by task: the odds of accepting a metaphor in the interpretation generation task were 3.24 times more than in the sensibility judgement task, as predicted. EoS had a positive effect on response decisions (i.e., easy simulation lead to more successful processing), which stayed constant between shallow and deep tasks. LDF also had a positive effect, and its positive interaction with task indicated that LDF had a larger effect on response decision in deep than shallow metaphor processing.

Response time. Results are shown in Table 9. Deep interpretation generation took significantly longer time than shallow sensibility judgement. EoS had opposite effects on acceptance versus rejection times, which was negative for 
"yes" responses but positive for "no" responses. Furthermore, EoS interacted with task, showing that its effect is larger for deep interpretation generation than for shallow sensibility judgement across both types of responses, as predicted by the depth of processing hypothesis. The effects of LDF were of the same pattern (despite producing effects in Experiment 1 but not in Experiment 2), though were not significant either as a main effect or in interaction with task. LDF interacted with EoS and task in a three-way interaction when response was "no", which resulted from the presence of an interaction between LDF and EoS in shallow processing but not in deep processing.

Summary. In summary, depth of processing affected the roles of both EoS and LDF. As predicted by the depth of processing hypothesis, EoS had a larger effect during deep processing than during shallow processing: specifying the meaning of a metaphor led people to rely more on embodied simulation than simply judging whether or not a metaphor makes sense. However, this greater reliance on the embodied component influenced only response times, and not the likelihood of accepting the metaphor as sensible or interpretable. Also as predicted, LDF had a larger effect on shallow processing than one deep processing, but only in terms of how it moderated the effect of EoS in "yes" response times: in successful metaphor processing, people relied on the linguistic shortcut to avoid unnecessarily effortful simulation more during sensibility judgement than during interpretation generation. While LDF also affected response decisions, the effect ran contrary to expectations by being larger for deep processing than for shallow processing: people relied on the linguistic shortcut more when attempting to generate a meaning for a metaphor than when deciding whether or not it made sense. Therefore, while our results supported the depth of processing hypothesis regarding EoS, the picture of more complex for LDF. 
Table 8

Logistic mixed-effect regression of response decision for cross-experiment comparison

\begin{tabular}{lrrr}
\hline & $b$ & $95 \%$ CI & $p$ \\
\hline Intercept & -0.25 & $-0.76-0.26$ & .337 \\
EoS & 1.48 & $1.36-1.60$ & $<.001 * * *$ \\
LDF & 0.11 & $0.02-0.21$ & $.023 *$ \\
Task & 1.18 & $0.50-1.86$ & $<.001 * * *$ \\
EoS x LDF & -0.00 & $-0.11-0.10$ & .926 \\
EoS x task & 0.01 & $-0.18-0.20$ & .919 \\
LDF x task & 0.22 & $0.06-0.38$ & $.006 * *$ \\
EoS x LDF x task & 0.00 & $-0.16-0.16$ & .979 \\
\hline
\end{tabular}

Note. $\dagger$ means $p<.10 ;{ }^{*}$ means $p<.05$;

** means $p<.01$; *** means $p<.001$.

$b$ is non-standardised regression coefficient.

Both predictors orthogonalized. 


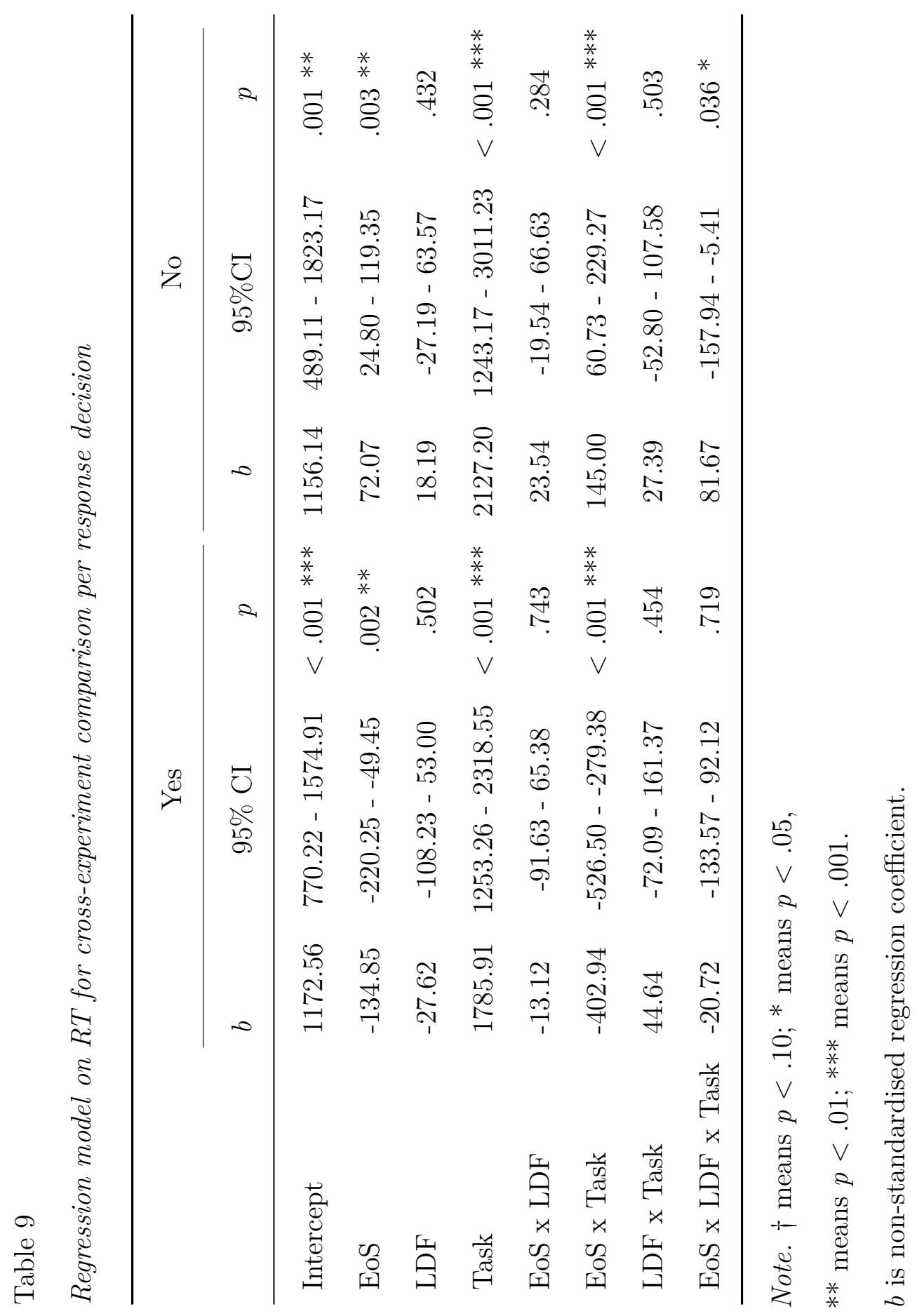




\section{General Discussion}

The current study investigates what makes metaphors easier to process from the perspective of grounded language comprehension (Barsalou et al., 2008; Connell \& Lynott, 2014b; Louwerse \& Jeuniaux, 2008; Vigliocco et al., 2009), with a view to establishing how the roles of the embodied component (i.e., simulation of sensorimotor, affective, and other experience) and linguistic component (i.e., distributional patterns of how words co-occur in language) varied with depth of processing. Our goal in taking this approach was to move the investigation of metaphor processing beyond the traditional factors of familiarity, conventionality, and aptness, which - while having a long history of use - have been increasingly criticized for theoretical and operational problems that limit their utility in explaining what makes one metaphor easier to understand than another. Following previous research (Connell \& Lynott, 2013; Louwerse \& Connell, 2011), we used LDF to operationalize processing in the linguistic component, which was calculated based on bi- to 5-gram co-occurrence frequencies from a large corpus. In addition, we proposed a new variable construct called EoS to operationalize processing in the embodied component (Liu et al., 2018), which reflected how easily people find it to arrive at a mental representation of metaphoric meaning.

Following our predictions, we found that EoS and LDF played unique and distinct roles in metaphor processing. They independently predicted the likelihood of accepting a metaphor in both shallow (Experiment 1) and deep (Experiment 2) processing tasks. People were more likely to decide a metaphor was sensible or interpretable if it was normally considered easy to simulate and if its words often appeared together in context. EoS could successfully predict the speed of processing in both tasks (i.e., people were faster to accept a metaphor as sensible or interpretable when it was easy to simulate, and faster to reject it as nonsensical or 
uninterpretable when it was difficult, regardless of LDF), and the effect was larger for deep interpretation generation than shallow sensibility judgement. In contrast, LDF only predicted the speed of processing in shallow sensibility judgement but not in deep interpretation generation. The more often a metaphor's words co-occurred in language, the faster people were to accept the metaphor when the depth of processing was shallow and good-enough processing (Ferreira et al., 2002) could suffice. Moreover, although LDF did not directly influence the speed of rejecting a metaphor as nonsensical, it moderated the effect of EoS by reducing its influence at low distributional frequencies. This pattern of effects supported the linguistic shortcut hypothesis that LDF can be used as a cognitive triage mechanism to identify metaphors that are unlikely to result in successful simulation, and so costly processing in the embodied component can be abandoned to avoid wasting resources, where task demands and processing goals allow (Connell, 2018; Connell \& Lynott, 2013, 2014b).

More broadly, results from the current study supports grounded views of language processing, in which the linguistic and the embodied components combine to form conceptual representations of meaning (Barsalou et al., 2008; Connell \& Lynott, 2014b; Louwerse \& Jeuniaux, 2008). Previous research has demonstrated the roles of the linguistic and embodied components in processing literal sentences about sensory features (Louwerse \& Connell, 2011), noun-noun conceptual combination (Connell \& Lynott, 2013), and property generation (Santos et al., 2011). However, the present paper shows for the first time that both components are also critical to processing metaphoric language.

Furthermore, these two components are activated at different speeds, where linguistic information reaches peak activation before simulated information (Barsalou et al., 2008), and is less costly, perfect for forming "good enough" representations (Louwerse \& Connell, 2011; Louwerse \& Jeuniaux, 2008). Of course, 
just because two words appear near one another does not necessarily mean that they were intended to form a metaphor. However, words tend to appear close together in language because their concepts form part of the same simulation of an idea or situation, which at least some of the times will be metaphorical. Conversely, words that seldom appear in the same context are unlikely to form a metaphor. As such, LDF represents a relatively coarse-grained, but nonetheless highly useful, approximation of whether a particular source and target can potentially form a metaphor. This idea is borne out by the results confirming the linguistic shortcut hypothesis. The linguistic component was found to influence the speed of acceptance in shallow processing, and even to reduce the effect of the embodied component when people rejected metaphors as nonsensical.

Our results are consistent with the conceptual combination study of Connell and Lynott (2013), which supported the cognitive triage mechanism of LDF. The critical differences between the conceptual combination study and the current one lies in that LDF had a smaller effect in our study than in the conceptual combination study. Specifically, in our study, LDF did not affect response times at all in the interpretation generation task, whereas in the conceptual combination study it had a significant positive effect on rejection time and overall response time in both tasks. One reason for these differences could be our addition of EoS as a predictor, independent from LDF. Specifically, we found in Experiment 1 that LDF was not entirely orthogonal to EoS, which means that both variables could account for a mutual portion of variance. Therefore, the LDF variable in the previous conceptual combination study did not only account for the linguistic component of conceptual representation, but may also have accounted partially for the embodied component. In contrast, the present study made sure that EoS and LDF were not only theoretically but also statistically distinct.

However, in contrast to the linguistic shortcut hypothesis, our study did not 
confirm the difference between shallow and deep processing in the cross-experiment analysis of the linguistic component (i.e., LDF did not interact with task), suggesting that LDF played roles of similar weights in shallow versus deep processing. One possible reason could be because the nature of the tasks did not particularly incentivize using the linguistic shortcut. Specifically, people had as much time as needed to make a response; they had unlimited time resource to form a mental representation using the embodied component and were not under pressure to conserve processing resources, and hence were not incentivized to employ the linguistic shortcut in an effort to avoid unnecessary costs. There is some support for this idea in the unexpected finding that LDF affected response decisions in deep processing more than in shallow processing. That is, when the potential cost of processing was higher (i.e., when people had to specify a meaning for a metaphor as opposed to just responding "yes" or "no"), people were more incentivized to avoid unnecessary processing and hence relied more on the linguistic component to guide their response decision. In future research, we plan to impose a time constraint on metaphor processing in order to further examine the linguistic shortcut hypothesis and the circumstances that incentivize its use.

A key motivation for adopting a grounded approach to metaphor processing is the problems and criticisms of traditional research the factors that have traditionally been the focus of metaphor processing (i.e. familiarity, conventionality, and aptness). As discussed in the introduction, the linguistic component (i.e., LDF calculated from a large corpus) and the embodied component (i.e., EoS measure) have several parallels with these factors. Specifically, LDF captures the extent to which familiarity, conventionality, and aptness are based on frequency of experience, and EoS captures the extent to which aptness is based on successful representation of metaphoric meaning. Therefore, EoS and LDF could subsume and account for the roles of the factors in traditional research. So could it be argued that our proposal 
only replaces the traditional factors with a couple of fancy-named variables without any theoretical contribution? We would argue not. The grounded approach we propose is more advantageous than the traditional approach for the following three reasons.

First, EoS and LDF are better operationalized. Both factors have clear definitions, unlike familiarity, conventionality, or aptness, which have different definitions to different research communities. EoS could only refer to how easy it is to simulate the meaning of the sentence. It takes the sentence as a whole, without selectively emphasizing the source or the target terms. In contrast, the definition of conventionality tends to focus on the source term (see Introduction), even though ratings of conventionality are context dependent (i.e. influenced by the target term). Also, the definition of LDF is objective and accurate. Unlike traditional familiarity or conventionality, which could concern either the metaphor sentence itself or the concept it refers to, LDF only refers to the co-occurrence of the constituent words. Thus, the operational definition of the two factors are unambiguous, improving the validity of the construct.

Second, we have demonstrated that these two predictors can exert statistically independent effects. Although the raw variables correlate to some degree, and there is evidence for net suppression in the models, it can be corrected by orthogonalizing the variables to remove the correlation between them. The grounded views of language processing state that the embodied and linguistic components are theoretically distinct, and our statistical treatment reflects just that. In other words, if our two predictors had the same core dependency as familiarity, conventionality and aptness, their effects would not have been unique and separable.

Finally, under the theoretical construct of the grounded views, the present studies produced findings that cannot be explained by the traditional factors, namely the interplay between EoS and LDF according to the required depth of processing. 
Previous studies have not considered metaphor processing as an interactive process that adapts to the situation. Based on the grounded view, we predicted that people would process metaphors using different strategies according to the requirement of the tasks. Our study found results that support this hypothesis. Indeed, previous studies which have shown an effect of aptness over conventionality often adopt a deep processing task, such as generating interpretations (Glucksberg \& Haught, 2006a; L. L. Jones \& Estes, 2006), listing attributes (Glucksberg \& Haught, 2006b), and creating new metaphors based on examples (Pierce \& Chiappe, 2008). Given the parallels we draw between aptness and EoS, this previous work is consistent with our finding that deeper processing relies more on EoS.

In conclusion, metaphor processing relies on conceptual representation that encompasses the two components proposed by the grounded account, that is the linguistic component and the embodied component. These two components interplay flexibly in order to produce representations that satisfy the requirement of the tasks. 


\section{References}

Allport, D. A. (1985). Distributed memory, modular subsystems and dysphasia. In S. Newman \& R. Epstein (Eds.), Current perspectives in dysphasia (pp. 207-244). Edinburgh: Churchill Livingstone.

Andrews, M., Vigliocco, G., \& Vinson, D. (2009). Integrating experiential and distributional data to learn semantic representations. Psychological Review, 116 (3), 463-498. doi:10.1037/a0016261

Aslin, R. N., Saffran, J. R., \& Newport, E. L. (1998). Computation of Conditional Probability Statistics By 8-Month-Old Infants. Psychological Science, 9(4), $321-324$.

Aust, F. (2016). Citr: 'RStudio' add-in to insert markdown citations. Retrieved from https://CRAN.R-project.org/package=citr

Aust, F., \& Barth, M. (2018). papaja: Create APA manuscripts with R Markdown. Retrieved from https://github.com/crsh/papaja

Bache, S. M., \& Wickham, H. (2014). Magrittr: A forward-pipe operator for $r$. Retrieved from https://CRAN.R-project.org/package=magrittr

Balota, D. A., Yap, M. J., Cortese, M. J., Hutchison, K. a, Kessler, B., Loftis, B., .. Treiman, R. (2007). The English Lexicon Project. Behavior Research Methods, 39(3), 445-59.

Barsalou, L. W. (1999). Perceptual symbol systems. The Behavioral and Brain Sciences, 22(4), 577-609; discussion 610-60.

Barsalou, L. W., Santos, A., Simmons, W. K., \& Wilson, C. (2008). Language and simulation in conceptual processing. In A. M. Glenberg \& A. C. Graesser (Eds.), Symbols, embodiment, and meaning (pp. 245-283). Oxford, England: 
Oxford University Press.

Bartoń, K. (2017). MuMIn: Multi-model inference. Retrieved from https://CRAN.R-project.org/package=MuMIn

Bates, D., \& Maechler, M. (2018). Matrix: Sparse and dense matrix classes and methods. Retrieved from https://CRAN.R-project.org/package=Matrix

Bates, D., Mächler, M., Bolker, B., \& Walker, S. (2015). Fitting linear mixed-effects models using lme4. Journal of Statistical Software, 67(1), 1-48. doi:10.18637/jss.v067.i01

Blasko, D. G., \& Connine, C. M. (1993). Effects of familiarity and aptness on metaphor processing. Journal of Experimental Psychology: Learning, Memory, and Cognition, 19(2), 295-308. doi:10.1037//0278-7393.19.2.295

Bonner, M. F., \& Grossman, M. (2012). Gray matter density of auditory association cortex relates to knowledge of sound concepts in primary progressive aphasia. Journal of Neuroscience, 32(23), 7986-7991. doi:10.1523/JNEUROSCI.6241-11.2012

Borghi, A. M., \& Binkofski, F. (2014). Words as Social Tools: An Embodied View on Abstract Concepts. New York, NY: Springer New York. doi:10.1007/978-1-4614-9539-0

Boroditsky, L. (2001). Does language shape thought? Mandarin and English speakers' conceptions of time. Cognitive Psychology, 43, 1-22. doi:10.1006/ cogp.2001.0748

Boulenger, V., Mechtouff, L., Thobois, S., Broussolle, E., Jeannerod, M., \& Nazir, T. A. (2008). Word processing in Parkinson's disease is impaired for action verbs but not for concrete nouns. Neuropsychologia, 46(2), 743-756. 
doi:10.1016/j.neuropsychologia.2007.10.007

Bowdle, B. F., \& Gentner, D. (2005). The career of metaphor. Psychological Review, 112(1), 193-216. doi:10.1037/0033-295X.112.1.193

Brants, T., \& Franz, A. (2006). Web 1T 5-gram Version 1 LDC2006T13. DVD. Philadelphia: Linguistic Data Consortium.

Campbell, S. J., \& Raney, G. E. (2015). A 25-year replication of Katz et al.'s (1988) metaphor norms. Behavior Research Methods, 48(1), 330-340. doi:10.3758/s13428-015-0575-2

Cardillo, E. R., Schmidt, G. L., Kranjec, A., \& Chatterjee, A. (2010). Stimulus design is an obstacle course: 560 matched literal and metaphorical sentences for testing neural hypotheses about metaphor. Behavior Research Methods, 42(3), 651-64. doi:10.3758/BRM.42.3.651

Cardillo, E. R., Watson, C. E., Schmidt, G. L., Kranjec, A., \& Chatterjee, A. (2012). From novel to familiar: tuning the brain for metaphors. NeuroImage, 59(4), 3212-21. doi:10.1016/j.neuroimage.2011.11.079

Chan, K. Q., Tong, E. M. W., Tan, D. H., \& Koh, A. H. Q. (2013). What do love and jealousy taste like? Emotion, 13(6), 1142-1149. doi:10.1037/a0033758

Chiappe, D. L., \& Kennedy, J. M. (1999). Aptness predicts preference for metaphors or similes, as well as recall bias. Psychonomic Bulletin 86 Review, 6(4), 668-76. doi:10.3758/BF03212977

Cohen, J., Cohen, P., West, S. G., \& Aiken, L. S. (2003). Applied Multiple Regression/Correlation Analysis for the Behavioral Sciences. Mahwah, NJ: Erlbaum.

Connell, L. (2018). What have labels ever done for us? The lingusitic shortcut in conceptual processing. Language, Cognition and Neuroscience. 
doi:10.1080/23273798.2018.1471512

Connell, L., \& Lynott, D. (2013). Flexible and fast: linguistic shortcut affects both shallow and deep conceptual processing. Psychonomic Bulletin $\&$ Review, 20(3), 542-50. doi:10.3758/s13423-012-0368-x

Connell, L., \& Lynott, D. (2014a). I See/Hear What You Mean: Semantic Activation in Visual Word Recognition Depends on Perceptual Attention. Journal of Experimental Psychology. General, 143(2), 527-533. doi:10.1037/a0034626

Connell, L., \& Lynott, D. (2014b). Principles of representation: Why you can't represent the same concept twice. Topics in Cognitive Science, 6(3), 390-406. doi:10.1111/tops.12097

Connell, L., \& Lynott, D. (2016). Do we know what we're simulating? Information loss on transferring unconscious perceptual simulation to conscious imagery. Journal of Experimental Psychology: Learning Memory and Cognition, 42(8), 1218-32. doi:10.1037/xlm0000245

Dowle, M., \& Srinivasan, A. (2018). Data.table: Extension of 'data.frame'. Retrieved from https://CRAN.R-project.org/package=data.table

Faust, M., \& Mashal, N. (2007). The role of the right cerebral hemisphere in processing novel metaphoric expressions taken from poetry: a divided visual field study. Neuropsychologia, 45(4), 860-70. doi:10.1016/j.neuropsychologia.2006.08.010

Ferreira, F., Bailey, K., \& Ferraro, V. (2002). Good-enough representations in language comprehension. Current Directions in Psychological Science, 11(1), $11-15$.

Gelman, A., \& Su, Y.-S. (2018). Arm: Data analysis using regression and multilevel/hierarchical models. Retrieved from 
https://CRAN.R-project.org/package=arm

Gentner, D., \& Wolff, P. (1997). Alignment in the processing of metaphor. Journal of Memory and Language, 37(3), 331-355. doi:10.1006/jmla.1997.2527

Giora, R. (1997). Understanding figurative and literal language: The graded salience hypothesis. Cognitive Linguistics, 8(3), 183-206.

Giora, R. (2007). Is metaphor special? Brain and Language, 100(2), 111-4. doi:10.1016/j.bandl.2006.08.001

Giora, R., \& Fein, O. (1999). On understanding familiar and less-familiar figurative language. Journal of Pragmatics, 31(12), 1601-1618. doi:10.1016/S0378-2166(99)00006-5

Glantz, S. A., \& Slinker, B. K. (1990). Primer of Applied Regression and Analysis of Variance (p. 777). McGraw-Hill Education.

Glenberg, A. M., \& Gallese, V. (2012). Action-based language: a theory of language acquisition, comprehension, and production. Cortex, 48(7), 905-22. doi:10.1016/j.cortex.2011.04.010

Glucksberg, S. (2003). The psycholinguistics of metaphor. Trends in Cognitive Sciences, 7(2), 92-96. doi:10.1016/S1364-6613(02)00040-2

Glucksberg, S., \& Haught, C. (2006a). Can Florida become like the next Florida? When metaphoric comparisons fail. Psychological Science, 17(11), 935-938. doi:10.1111/j.1467-9280.2006.01807.x

Glucksberg, S., \& Haught, C. (2006b). On the Relation Between Metaphor and Simile: When Comparison Fails. Mind and Language, 21(3), 360-378. doi:10.1111/j.1468-0017.2006.00282.x

Glucksberg, S., Gildea, P., \& Bookin, H. (1982). On understanding nonliteral speech: Can people ignore metaphors? Journal of Verbal Learning and Verbal 
Behavior, (21), 85-98.

Goldberg, R. F., Perfetti, C. A., \& Schneider, W. (2006). Perceptual Knowledge Retrieval Activates Sensory Brain Regions. Journal of Neuroscience, 26(18), 4917-4921. doi:10.1523/JNEUROSCI.5389-05.2006

Haught, C. (2014). Spain is Not Greece: How Metaphors are Understood. Journal of Psycholinguistic Research, 43(4), 351-356. doi:10.1007/s10936-013-9258-2

Hauk, O., Johnsrude, I., \& Pulvermüller, F. (2004). Somatotopic Representation of Action Words in Human Motor and Premotor Cortex. Neuron, 41(2), 301-307. doi:10.1016/S0896-6273(03)00838-9

Henry, L., \& Wickham, H. (2018). Purrr: Functional programming tools. Retrieved from https://CRAN.R-project.org/package=purrr

Johns, B. T., \& Jones, M. N. (2012). Perceptual Inference Through Global Lexical Similarity. Topics in Cognitive Science, 4(1), 103-120. doi:10.1111/j.1756-8765.2011.01176.x

Jones, L. L., \& Estes, Z. (2005). Metaphor comprehension as attributive categorization. Journal of Memory and Language, 53(1), 110-124. doi:10.1016/j.jml.2005.01.016

Jones, L. L., \& Estes, Z. (2006). Roosters, robins, and alarm clocks: Aptness and conventionality in metaphor comprehension. Journal of Memory and Language, 55(1), 18-32. doi:10.1016/j.jml.2006.02.004

Katz, A. N., Paivio, A., Marshark, M., \& Clark, J. M. (1988). Norms for 204 literary and 260 nonliterary metaphors on 10 psychological dimensions. Metaphor and Symbolic Activity, 3(4), 191-214.

Kirkham, N. Z., Slemmer, J. A., \& Johnson, S. P. (2002). Visual statistical learning in infancy: Evidence for a domain general learning mechanism. Cognition, 
83(2), 4-5. doi:10.1016/S0010-0277(02)00004-5

Kuznetsova, A., Brockhoff, P. B., \& Christensen, R. H. B. (2017). lmerTest package: Tests in linear mixed effects models. Journal of Statistical Software, 82(13), 1-26. doi:10.18637/jss.v082.i13

Lai, V. T., \& Curran, T. (2013). ERP evidence for conceptual mappings and comparison processes during the comprehension of conventional and novel metaphors. Brain and Language, 127(3), 484-496.

doi:10.1016/j.bandl.2013.09.010

Lai, V. T., Curran, T., \& Menn, L. (2009). Comprehending conventional and novel metaphors: An ERP study. Brain Research, 1284, 145-155. doi:10.1016/j.brainres.2009.05.088

Lakoff, G., \& Johnson, M. (1999). Philosophy in the Flesh: The Embodied Mind and Its Challenge to Western Thought. Basic Books.

Laurent, J. P., Denhières, G., Passerieux, C., Iakimova, G., \& Hardy-Baylé, M. C. (2006). On understanding idiomatic language: The salience hypothesis assessed by ERPs. Brain Research, 1068(1), 151-160. doi:10.1016/j.brainres.2005.10.076

Liu, P. Q., Connell, L., \& Lynott, D. (2018). Ease-of-Simulation norms for 452 Adjective Metaphors. Manuscript Under Review.

Louwerse, M. M., \& Connell, L. (2011). A taste of words: linguistic context and perceptual simulation predict the modality of words. Cognitive Science, 35(2), 381-98. doi:10.1111/j.1551-6709.2010.01157.x

Louwerse, M. M., \& Hutchinson, S. (2012). Neurological evidence linguistic processes precede perceptual simulation in conceptual processing. Frontiers 
in Psychology, 3(October), 385. doi:10.3389/fpsyg.2012.00385

Louwerse, M. M., \& Jeuniaux, P. (2008). Language comprehension is both embodied and symbolic. In A. Glenberg \& A. C. Graesser (Eds.), Embodiment and meaning: A debate (pp. 309-326). Oxford, England: Oxford University Press.

Louwerse, M. M., Hutchinson, S., Tillman, R., \& Recchia, G. (2015). Effect size matters: the role of language statistics and perceptual simulation in conceptual processing. Language, Cognition and Neuroscience, 30(4), 430-447. doi:10.1080/23273798.2014.981552

Lynott, D., \& Connell, L. (2009). Modality exclusivity norms for 423 object properties. Behavior Research Methods, 41(2), 558-64. doi:10.3758/BRM.41.2.558

Lynott, D., \& Connell, L. (2010). Embodied conceptual combination. Frontiers in Psychology, 1 (November), 212. doi:10.3389/fpsyg.2010.00212

Mashal, N. (2013). The role of working memory in the comprehension of unfamiliar and familiar metaphors. Language and Cognition, 5(4), 409-436. doi:10.1515/langcog-2013-0024

Mashal, N., Faust, M., \& Hendler, T. (2005). The role of the right hemisphere in processing nonsalient metaphorical meanings: application of principal components analysis to fMRI data. Neuropsychologia, 43(14), 2084-100. doi:10.1016/j.neuropsychologia.2005.03.019

Morey, R. D., \& Rouder, J. N. (2018). BayesFactor: Computation of bayes factors for common designs. Retrieved from https://CRAN.R-project.org/package=BayesFactor

Niedenthal, P. M. (2007). Embodying Emotion. Science, 316(5827), 1002-1005. 
doi:10.1126/science.1136930

Nowosad, J. (2018). Rcartocolor: 'CARTOColors' palettes. Retrieved from https://CRAN.R-project.org/package=rcartocolor

Peleg, O., Giora, R., \& Fein, O. (2001). Salience and Context Effects: Two Are Better Than One. Metaphor and Symbol, 16(3-4), 173-192. doi:10.1080/10926488.2001.9678894

Pierce, R. S., \& Chiappe, D. L. (2008). The Roles of Aptness, Conventionality, and Working Memory in the Production of Metaphors and Similes. Metaphor and Symbol, 24(1), 1-19. doi:10.1080/10926480802568422

Pinheiro, J., Bates, D., DebRoy, S., Sarkar, D., \& R Core Team. (2018). nlme: Linear and nonlinear mixed effects models. Retrieved from https://CRAN.R-project.org/package=nlme

Plate, T., \& Heiberger, R. (2016). Abind: Combine multidimensional arrays. Retrieved from https://CRAN.R-project.org/package=abind

Plummer, M., Best, N., Cowles, K., \& Vines, K. (2006). CODA: Convergence diagnosis and output analysis for mcmc. $R$ News, 6(1), 7-11. Retrieved from https://journal.r-project.org/archive/

R Core Team. (2018). R: A language and environment for statistical computing. Vienna, Austria: R Foundation for Statistical Computing. Retrieved from https://www.R-project.org/

Revelle, W. (2018). Psych: Procedures for psychological, psychometric, and personality research. Evanston, Illinois: Northwestern University. Retrieved from https://CRAN.R-project.org/package=psych

Riordan, B., \& Jones, M. N. (2011). Redundancy in perceptual and linguistic experience: Comparing feature-based and distributional models of semantic 
representation. Topics in Cognitive Science, 3(2), 303-345.

doi:10.1111/j.1756-8765.2010.01111.x

Robinson, D. (2018). Broom: Convert statistical analysis objects into tidy data frames. Retrieved from https://CRAN.R-project.org/package=broom

Roncero, C., \& Almeida, R. G. de. (2014a). Semantic properties, aptness, familiarity, conventionality, and interpretive diversity scores for 84 metaphors and similes. Behavior Research Methods. doi:10.3758/s13428-014-0502-y

Roncero, C., \& Almeida, R. G. de. (2014b). The importance of being apt: metaphor comprehension in Alzheimer's disease. Frontiers in Human Neuroscience, 8, 973. doi:10.3389/fnhum.2014.00973

Santos, A., Chaigneau, S. E., Simmons, W. K., \& Barsalou, L. W. (2011). Property generation reflects word association and situated simulation. Language and Cognition, 3111, 83-119. doi:10.1515/

Simmons, W. K., Ramjee, V., Beauchamp, M. S., McRae, K., Marin, A., \& Barsalou, L. W. (2007). A common neural substrate for perceiving and knowing about color. Neuropsychologia, 45(12), 2802-2810. doi:10.1016/j.neuropsychologia.2007.05.002.A

Solt, F., \& Hu, Y. (2015). interplot: Plot the effects of variables in interaction terms. Available at The Comprehensive R Archive Network (CRAN). Retrieved from https://CRAN.R-project.org/package=interplot

Thibodeau, P. H., \& Durgin, F. H. (2011). Metaphor Aptness and Conventionality: A Processing Fluency Account. Metaphor and Symbol, 26(November), 206-226. doi:10.1080/10926488.2011.583196

Venables, W. N., \& Ripley, B. D. (2002). Modern applied statistics with s (Fourth.). 
New York: Springer. Retrieved from http://www.stats.ox.ac.uk/pub/MASS4

Vigliocco, G., Meteyard, L., Andrews, M., \& Kousta, S. (2009). Toward a theory of semantic representation. Language and Cognition, 1(2), 219-247. doi:10.1515/LANGCOG.2009.011

Wickham, H. (2009). Ggplot2: Elegant graphics for data analysis. Springer-Verlag New York. Retrieved from http://ggplot2.org

Xie, Y. (2015). Dynamic documents with $R$ and knitr (2nd ed.). Boca Raton, Florida: Chapman; Hall/CRC. Retrieved from https://yihui.name/knitr/

Xie, Y. (2016). Bookdown: Authoring books and technical documents with $R$ markdown. Boca Raton, Florida: Chapman; Hall/CRC. Retrieved from https://github.com/rstudio/bookdown 